\title{
Degradation of Modulation and Noise Characteristics of Semiconductor Lasers After Propagation in Optical Fiber Due to a Phase Shift Induced by Stimulated Brillouin Scattering
}

\author{
Eva Peral and Amnon Yariv, Life Fellow, IEEE
}

\begin{abstract}
Here we demonstrate theoretically that stimulated Brillouin scattering (SBS) can induce a phase shift of the optical carrier relative to its sidebands due to the waveguiding effect of the optical fiber on the acoustic wave. This causes conversion of frequency modulation to intensity modulation, which results in an increase in the relative intensity noise and degradation of the modulation response of directly modulated lasers after propagation in an optical fiber, in agreement with our experimental observations. Suppression of SBS can be achieved at low frequencies and high modulation powers due to the laser adiabatic chirp.
\end{abstract}

Index Terms - Brillouin scattering, laser noise, optical fiber communication, optical fiber measurement, optical modulation, optical propagation in nonlinear media, semiconductor lasers.

\section{INTRODUCTION}

$\mathbf{F}$ IBER nonlinearities may significantly degrade the performance of high-speed lightwave systems using highpower laser sources [1]. In particular, it has been shown through signal-to-noise ratio (SNR) and bit-error rate (BER) measurements that the onset of stimulated Brillouin scattering (SBS) can be a major impairment in directly modulated fiberoptic systems [2], [3].

Previous studies emphasized degradations resulting from power saturation [4], spontaneous Brillouin scattering noise [5], and the effect of the backward-propagating signal on the laser source [1]. Here, we provide experimental evidence of an SBS-induced phase shift of the optical carrier relative to its sidebands that leads to distortion of the modulation response (MR) and an increase in relative intensity noise (RIN) at low frequencies due to conversion of laser frequency modulation (FM), i.e., laser chirp, to intensity modulation (IM). This phase shift is explained theoretically by taking into consideration the guided nature of the acoustic waves in optical fibers.

Manuscript received December 7, 1998; revised April 7, 1999. This work was supported by the Defense Advanced Research Projects Agency, the Office of Naval Research, and the Air Force Office of Scientific Research under Award N00014-91-J-1195.

The authors are with the Department of Applied Physics, California Institute of Technology, Pasadena, CA 91125 USA.

Publisher Item Identifier S 0018-9197(99)05951-5.
In an optical fiber, the acoustooptic interaction between sound and optical waves differs from the Brillouin scattering in a bulk medium [6]. In a fiber, a large number of (longitudinal) acoustic modes are excited by the light, which are the eigenmodes of the cylindrical structure rather than plane waves, and this leads to a relaxation of the wavevector selection rule. As a consequence, spontaneous Brillouin scattering in the forward direction was observed and was called guided acoustic wave Brillouin scattering [6]. It has been recently found that this effect can contribute to timing jitter in soliton transmission systems [7] and affect propagation of NRZ pulse trains [8].

Here, we show that the guided nature of the acoustic waves in optical fibers can also affect backward SBS, inducing a phase shift of the optical waves. In order that a significant acoustooptic interaction occurs, not only do the propagation constants have to be phase matched, but there also has to be a significant overlap between the transverse profiles of acoustic and optical modes. The latter condition is satisfied for modes with phase velocity close to but greater than the longitudinal sound velocity $v_{l}$. However, if the phase velocity is less than $v_{l}$, the acoustic mode becomes a surface wave with maximum amplitude near the surface of the cylinder and will be hardly excited by the optical wave. It will be shown that this results in a slight asymmetry in the Brillouin gain spectrum, which can be observed in precise measurements of Brillouin gain spectra (see, e.g., [9, Fig. 4(a)]), and a phase shift of the optical waves affected by SBS, in agreement with our experimental results. If most of the optical power is contained in the carrier and the modulation frequency is larger than the Brillouin gain bandwidth, only the carrier undergoes SBS and thus suffers an additional phase shift relative to its sidebands. As a consequence, conversion of FM to IM occurs, which is experimentally observed in measurements of MR and RIN.

This paper is structured as follows. Section II describes the experimental setup that was used to measure the RIN and MR. In Section III, the effect of fiber nonlinearities on MR is studied. We show experimental evidence that SBS induces a phase change in the optical carrier with respect to the sidebands that leads to an increase of the MR at low frequencies due to FM-to-IM conversion. Section IV presents the effect of SBS on RIN. The SBS-induced phase 


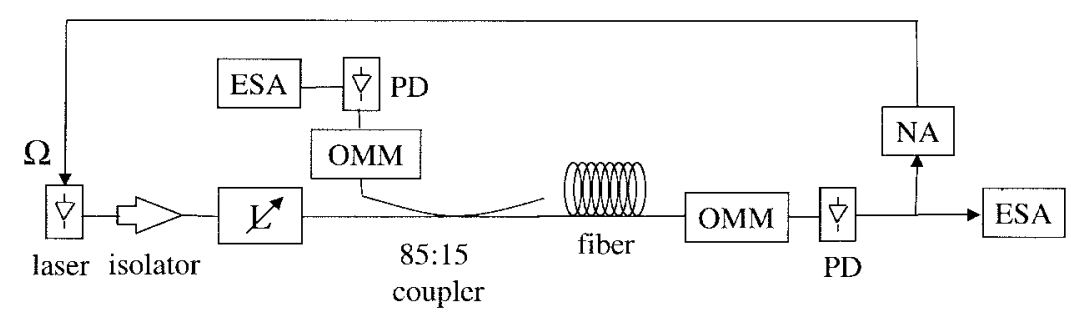

Fig. 1. Experimental setup. $L$ : variable optical attenuator; OMM: optical multimeter; PD: photodetector; NA: network analyzer; ESA: electrical spectrum analyzer.

change causes an increase in RIN at low frequencies by conversion of phase noise to intensity noise. In addition, it is shown that SBS causes excess noise at frequencies around harmonics of the acoustic wave frequency for both forward and backward propagating waves. Section V contains a theory of the acoustooptic interaction, including the waveguiding effect of the optical fiber on the acoustic wave. It is shown that SBS induces a phase shift of the optical carrier relative to its sidebands, which satisfactorily explains the measured increase in RIN and MR at low frequencies. Finally, in Section VI, the main conclusions are outlined.

\section{EXPERIMENTAL SET-UP}

The diagram of the experimental setup is shown in Fig. 1. The optical source was a high-power MQW-DFB laser (ORTEL Corporation) operating at $1.55 \mu \mathrm{m}$. This laser was biased at $300 \mathrm{~mA}$, yielding an output power at the laser pigtail of $14.4 \mathrm{dBm}$, and had a threshold of $18 \mathrm{~mA}$. The laser was directly modulated with the signal from a network analyzer (NA) using a microwave probe. The laser light was attenuated and launched into an $85 / 15 \%$ fiber directional coupler. The $85 \%$ port was connected onto a spool of standard telecommunications fiber (from 25 to $75 \mathrm{~km}$ ), the output signal was photodetected and then the MR and RIN were measured with a NA and an electrical spectrum analyzer (ESA), respectively. Angled connectors were used to minimize reflections.

Measurements of MR and RIN before and after propagation in dispersive fiber, with the signal output attenuated to avoid nonlinear effects, were used to determine laser parameters [10], [11], such as resonance frequency $\Omega_{0}=14 \mathrm{GHz}$, damping factor $\gamma_{0}=16 \mathrm{GHz}$, linewidth enhancement factor $\alpha=-4.6$, photon lifetime $\tau_{\mathrm{ph}}=10 \mathrm{ps}$, carrier lifetime $\tau=0.12$ ns, linewidth $0.5 \mathrm{MHz}$, and the fiber dispersion parameter $\beta^{\prime \prime}=-20 \mathrm{ps}^{2} / \mathrm{km}$.

The amount of optical power launched into the fiber was controlled with a variable optical attenuator, and measurements of RIN and MR were performed at several optical powers and modulation powers for the forward and backward propagating fields.

\section{EFFECT OF SBS on Modulation Response}

Semiconductor lasers exhibit chirp, i.e., the frequency of the optical field is modulated whenever the intensity is modulated. Thus, the complex electric field amplitude $\tilde{E}$ at the output of a semiconductor laser directly modulated with a modulation frequency $\Omega / 2 \pi$ can be expressed in the form

$$
\begin{aligned}
\tilde{E}= & P_{0}^{1 / 2}\left[1+m_{\mathrm{IM}} \cos \left(\Omega t+\varphi_{\mathrm{IM}}\right)\right]^{1 / 2} \\
& \cdot \exp \left[i m_{\mathrm{FM}} \sin \left(\Omega t+\varphi_{\mathrm{FM}}\right)\right] \\
= & P_{0}^{1 / 2}\left[1+\left(\frac{\Delta P}{P_{0}} \exp (i \Omega t)+\text { c.c. }\right)\right]^{1 / 2} \\
& \cdot \exp [i(\Delta \phi \exp (i \Omega t)+\text { c.c. })]
\end{aligned}
$$

where $m_{\mathrm{IM}}$ and $m_{\mathrm{FM}}$ are the IM and FM indices, respectively. The ratio $\Delta \phi /\left(\Delta P / P_{0}\right)=\left(m_{\mathrm{FM}} / i m_{\mathrm{IM}}\right) e^{i \Delta \varphi}$, where $\Delta \varphi=$ $\varphi_{\mathrm{FM}}-\varphi_{\mathrm{IM}}$ is the dephasing angle between the laser frequency and intensity modulation, will be referred to in what follows as the phase-to-intensity (modulation index) ratio (PIR), which for light produced by a semiconductor laser is a function of the modulation frequency $\Omega$. In the case of direct modulation of the laser with modulation current $\Delta I_{m}$, the PIR can be expressed as

$$
\left.\left.\mathrm{PIR}\right|_{\Delta I_{m}} \equiv \frac{\Delta \phi}{\Delta P / P_{0}}\right|_{\Delta I_{m}}=-\frac{\alpha}{2}\left(1+\frac{\kappa(\Omega)}{i \Omega}\right)
$$

where $\alpha$ is the linewidth enhancement factor and $\kappa(\Omega)$ is related to several laser parameters and contributes to a quasiadiabatic chirp [10].

Due to group velocity dispersion, linear propagation in dispersive optical fiber produces a phase change of the sidebands relative to the optical carrier, which results in partial conversion of FM into IM, which is photodetected at the fiber output [11], [12]. At low modulation frequencies, the dispersion is very small and the change in MR after propagation is simply given by the fiber loss. However, our experimental results show that, as the optical power launched in the fiber increases, some optical power is backreflected by SBS, and, surprisingly, correlated with this power loss, the MR at low frequencies increases. We attribute this increase to a phase change in the optical carrier induced by SBS. At high $\Omega$, this small nonlinear phase change can be neglected compared to that resulting from dispersion. However, at low $\Omega$, it becomes dominant and, as a consequence, the large adiabatic chirp that semiconductor lasers present at low $\Omega$ is converted into intensity modulation. The origin of this phase change will be explained in Section V.

Let $\theta(\Omega, z)$ be the dephasing between the optical carrier and the optical sideband at $\Omega$ due to both fiber dispersion and fiber nonlinearity, that is,

$$
\begin{aligned}
\theta(\Omega, z) & =\theta_{\mathrm{disp}}(\Omega, z)+\theta_{\mathrm{NL}}(\Omega, z) \\
& =\frac{1}{2} \beta^{\prime \prime} \Omega^{2} z+\theta_{\mathrm{NL}}(\Omega, z) .
\end{aligned}
$$




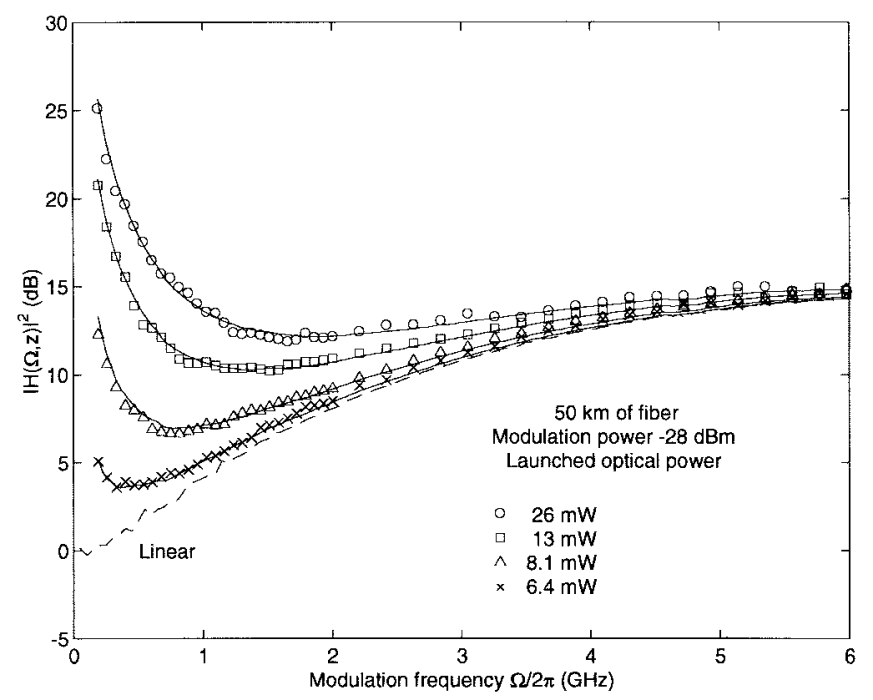

Fig. 2. Change in modulation response $|H(\Omega, z)|^{2}$ for $50 \mathrm{~km}$ of fiber, -28 $\mathrm{dBm}$ modulation power, and several launched powers into the fiber. Dashed line is measured $|H(\Omega, z)|^{2}$ in the absence of nonlinear effects. Solid line is theory.

Then, the detected photocurrent at a small-signal modulation frequency $\Omega$ at the fiber output $\Delta I(\Omega, z)$ is related to that at the laser output $\Delta I(\Omega, 0)$ by

$$
\begin{aligned}
H(\Omega, z) & \equiv\left[L_{\mathrm{DC}}(z) L(\Omega, z)\right]^{1 / 2} \frac{\Delta I(\Omega, z)}{\Delta I(\Omega, 0)} \\
& =\cos \theta-2 \sin \theta \operatorname{PIR}(\Omega) .
\end{aligned}
$$

The electrical transfer function $H(\Omega, z)$ is normalized by the loss in the fiber, where $L_{\mathrm{DC}}(z)$ and $L(\Omega, z)$ are the power loss suffered by the optical carrier and the sideband, respectively, due to both fiber attenuation and SBS.

Since the spectrum of the Brillouin gain is very narrow ( $\sim 15 \mathrm{MHz}$, as shown in Section IV), it can be assumed that for the modulation range considered here, $\Omega>50 \mathrm{MHz}$, no cross interactions between the optical carrier and sidebands contribute to the SBS gain. In addition, in the small-signal regime, only two modulation sidebands need be taken into account. Since most of the optical power is contained in the carrier, we can assume that the sidebands only suffer linear fiber loss, whereas part of the power in the carrier is lost to SBS. Thus, we set

$$
\begin{aligned}
L(\Omega, z) & =L_{\operatorname{Lin}}(z)=e^{-\alpha z} \\
L_{\mathrm{DC}} & =L_{\operatorname{Lin}} L_{\mathrm{NL}} .
\end{aligned}
$$

Fig. 2 shows $|H(\Omega, z)|^{2}$ for a small modulation power and several optical input powers. At low modulation frequencies, the laser chirp is dominated by the quasi-adiabatic term and varies as $1 / \Omega$. A negative $\theta_{\mathrm{NL}}$ results in part of this chirp converted into IM, and $|H(\Omega, z)|^{2}$ increases with decreasing $\Omega$ as $1 / \Omega$. At high $\Omega$, and using the normalization explained above, $|H(\Omega, z)|^{2}$ approaches its linear regime value.

Since the modulation power is very small $\left(m_{\mathrm{IM}} \approx 0.08 \%\right)$, the small-signal approximation is valid and (4)-(6) can be used to determine the nonlinear phase change $\theta_{\mathrm{NL}}$. The PIR was measured by first attenuating the power launched in the fiber so that the linear theory holds using the method developed in [10]. We see that the theory above accounts well for the experimental results. Fig. 3 shows the measured $\theta_{\mathrm{NL}}$ together with the backscattered and forward output powers as a function of the optical input power. The dotted line is the predicted $\theta_{\mathrm{NL}}$ using the theory in Section V.

At higher modulation powers, but low enough so that $m_{\mathrm{IM}}$ is less than $2 \%$, even though the small-signal theory would still be valid in the linear regime, it fails to predict the measured MR after propagation in fiber at high optical powers. As the modulation power is raised, the optical bandwidth increases due to a larger laser chirp, and the SBS is reduced. The consequence of this is twofold. First, since the FM index $m_{\mathrm{FM}}$ is a function of $\Omega$, the detected dc photocurrent fluctuates as $\Omega$ varies. Second, the MR at sufficiently low $\Omega$ and high modulation power returns to that in the linear regime.

Here we present a large-signal model that explains the observed phenomena. As the laser chirp increases, the optical power is distributed among an increasing number of FM sidebands with weights $c_{n}$ such as

$$
\begin{aligned}
\tilde{E}= & P_{0}^{1 / 2} \sum_{n} c_{n} e^{\mathrm{in}\left(\Omega t+\varphi_{\mathrm{FM}}\right)} \\
c_{n}(z=0)= & J_{n}\left(m_{\mathrm{FM}}\right)+\frac{m_{\mathrm{IM}}}{4}\left(J_{n+1}\left(m_{\mathrm{FM}}\right) e^{i \Delta \varphi}\right. \\
& \left.+J_{n-1}\left(m_{\mathrm{FM}}\right) e^{-i \Delta \varphi}\right)
\end{aligned}
$$

where $J_{n}$ are Bessel functions and we used the fact that $m_{\mathrm{IM}}$ is small. Thus, after propagation in fiber, the coefficients $c_{n}$ are given by

$$
c_{n}(z)=\sqrt{L(n \Omega, z)} e^{i \theta(n \Omega, z)} c_{n}(0) .
$$

The detected dc photocurrent $I_{\mathrm{DC}}$ can be obtained as

$$
I_{\mathrm{DC}}(z)=R(0) P_{0} \sum_{n} c_{n}(z) c_{n}^{*}(z)
$$

where $R(\Omega)$ is the photodiode responsivity, and the photocurrent at $\Omega$ is given by

$$
\Delta I(\Omega, z)=R(\Omega) P_{0} \sum_{n} c_{n+1}(z) c_{n}^{*}(z)
$$

Fig. 4 shows the measured $|H(\Omega, z)|^{2}$ and received power for several modulation powers. In normalizing $|H(\Omega, z)|^{2}$, we have assumed for convenience, since $L(\Omega, z)$ is difficult to measure, that the modulation sidebands only suffer linear fiber loss, i.e., we set $H(\Omega, z) \equiv$ $\left(L L_{L \text { in }}\right)^{1 / 2}[\Delta I(\Omega, z) / \Delta I(\Omega, 0)]$. This is not a valid assumption at large modulation powers and low $\Omega$, i.e., for large laser chirp. In fact, when $J_{0}\left(m_{\mathrm{FM}}\right) \approx 0$, most of the optical power is contained in the first sideband, which is then partly backscattered by SBS, and $L(\Omega, z)>L_{L \text { in }}$. As a consequence of the normalization we used, $|H(\Omega, z)|^{2}$ becomes negative (in decibels). As the bandwidth of the signal increases, the SBS is reduced, and the received power rises. The ripple in $|H(\Omega, z)|^{2}$ and received power at low frequencies is due to the oscillatory behavior of the Bessel functions. On the contrary, the structure observed in the received power at $\Omega \sim 0.7 \mathrm{GHz}$ 


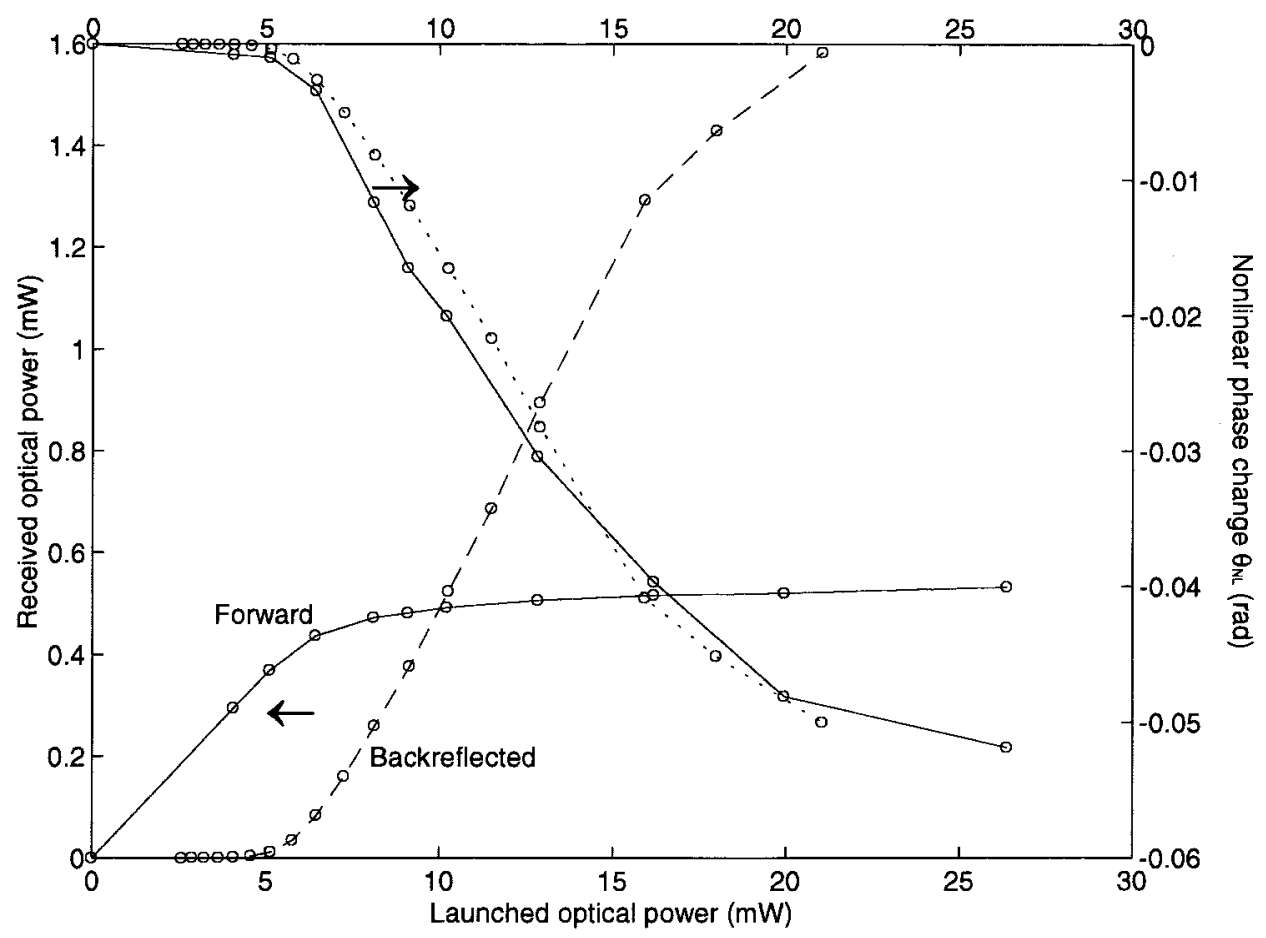

Fig. 3. Left axis: forward (solid) and backscattered (dashed) optical powers for $50 \mathrm{~km}$ of fiber. Right axis: nonlinear phase change (solid is experimental, dotted is theoretical prediction as described in text).

is due to the frequency response of the microwave probe employed to modulate the laser.

The large-signal theory described above was used to explain the experimental data and is represented in Fig. 4 with a solid trace. To determine $L(n \Omega, z)$ and $(n \Omega, z)$, we neglect, as we did in the small-signal case, cross interaction between sidebands. Then, we can assume that the power loss and phase change in each FM sideband can be extracted from the previous data in Fig. 3, for the given power in the FM band. Good agreement has been found between this simple model and the measurement. The main discrepancy occurs when $|H(\Omega, z)|^{2}$ becomes negative in decibels, which our model underestimates. This is attributed to the assumption of no cross interaction.

The inset in Fig. 4 shows $|H(\Omega, z)|^{2}$ in an extended frequency range for both linear and nonlinear regimes. It is observed that at high $\Omega$, and with the normalization in (4)-(6), both curves coincide. Thus, the effect of self-phase-modulation (SPM) that was recently reported in [13] does not appear in our data, which indicates that SBS manifests itself at lower optical powers than SPM.

\section{EFFECT OF SBS on Relative InTENSITy NoISE}

The presence of SBS alters the RIN in two ways. First, excess noise appears around $0 \mathrm{~Hz}$ and around harmonics of the acoustic wave frequency for both backward and forward propagating fields [14]. Second, we show here that the SBS-induced phase change in the optical carrier results in conversion of part of the FM noise into IM noise, which causes additional excess noise at low frequencies.

The FM-to-IM conversion of laser noise can be treated in a way similar to the case of MR in Section III. The PIR due to photon fluctuations, which are generated by a Langevin noise source $F_{P}$, is given by

$$
\left.\left.\operatorname{PIR}\right|_{F_{P}} \equiv \frac{\Delta \phi}{\Delta P / P}\right|_{F_{P}}=-\frac{\alpha}{2} \frac{\Omega_{0}^{2}}{\Omega^{2}} \frac{1+i \Omega \tau_{p h}}{1+1 /(i \Omega \tau)} .
$$

As $\Omega$ decreases, the PIR increases, and a small phase change of the optical carrier can result in a strong enhancement of the noise. This is observed in Fig. 5, where the RIN is plotted for several launched powers in the fiber. Equations (4)-(6) together with (12) can be used to determine $\theta_{\mathrm{NL}}$, which is shown in the inset. The slight discrepancy between experiment and theory is attributed to the simple model used to describe the RIN, which does not include the effect of side modes [15] and FM-to-IM noise conversion due to double Rayleigh scattering [16]. These two effects are independent of the launched power and explain why $\theta_{\mathrm{NL}}$ does not equal exactly zero at small launched powers. The excess noise induced by SBS near $0 \mathrm{~Hz}$ reported in [14] would affect frequencies below the ones here considered and does not need to be included in the analysis.

The RIN spectrum of the forward (backward) wave exhibits excess noise around harmonics of $\Omega_{B}$ due to mixing upon detection of the incident (Stokes) wave with small reflections of the Stokes (incident) wave caused by Rayleigh scattering [14]. When the laser is modulated at a frequency $\Omega$, SBS induces additional excess noise at frequencies $m \Omega_{B}+n \Omega$, with $m$ and $n$ integers. Fig. 6 displays the measured RIN of the forward and backward fields after propagation in 75 $\mathrm{km}$ of fiber for frequencies around $0 \mathrm{~Hz}$ and $\Omega_{B}$. The power launched into the fiber was $13 \mathrm{dBm}$ and the laser was directly modulated at $300 \mathrm{MHz}$ with several modulation powers. As can be observed in Fig. 6(a), the excess noise due to FM- 


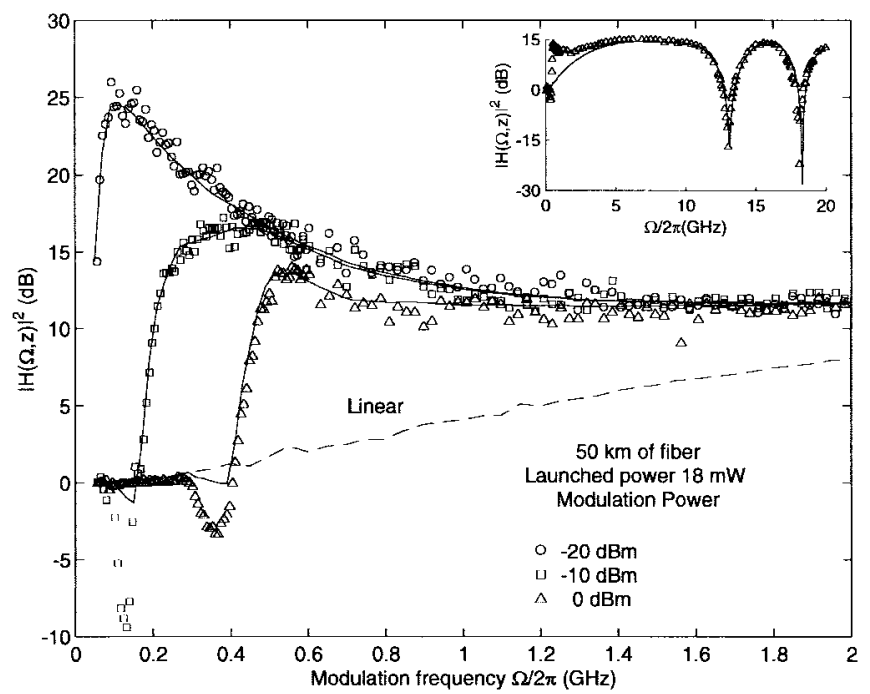

(a)

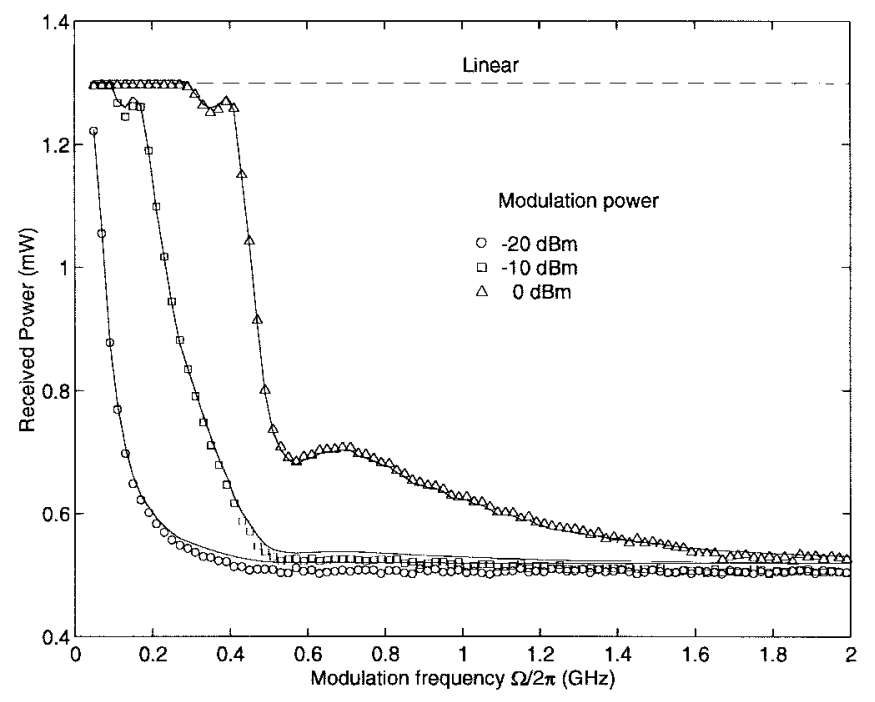

(b)

Fig. 4. (a) Change in modulation response $|H(\Omega, z)|^{2}$. (b) Received power as function of modulation frequency for $50 \mathrm{~km}$ of fiber, $18 \mathrm{~mW}$ of launched optical power, and several modulation powers. Dashed line: measured data in the absence of nonlinear effects. Solid line: theory. The inset in (a) shows $|H(\Omega, z)|^{2}$ in an extended frequency range for $18-\mathrm{mW}$ optical power (triangles) and linear regime (solid) with $0-\mathrm{dBm}$ modulation power.

to-IM conversion also appears at harmonics of the modulation frequency $n \Omega$. As discussed in Section III, for sufficiently high modulation power and low modulation frequency, the input optical power is distributed in many sidebands, and the SBS, and consequently the excess noise, is suppressed.

The RIN spectrum of the backward Stokes wave at $0 \mathrm{~Hz}$ [Fig. 6(c)] can be used to determine the spectrum of the Brillouin gain [17] and gives a rough estimate of the Brillouin bandwidth to be around $15 \mathrm{MHz}$. The measured RIN level of the Stokes wave was approximately $-80 \mathrm{~dB} / \mathrm{Hz}$ (irrespective of fiber length and launched power), which can be related to the strength of the Langevin noise source that describes the thermal excitation of acoustic waves [18]. Residual excess noise at harmonics of the modulation frequency is due to

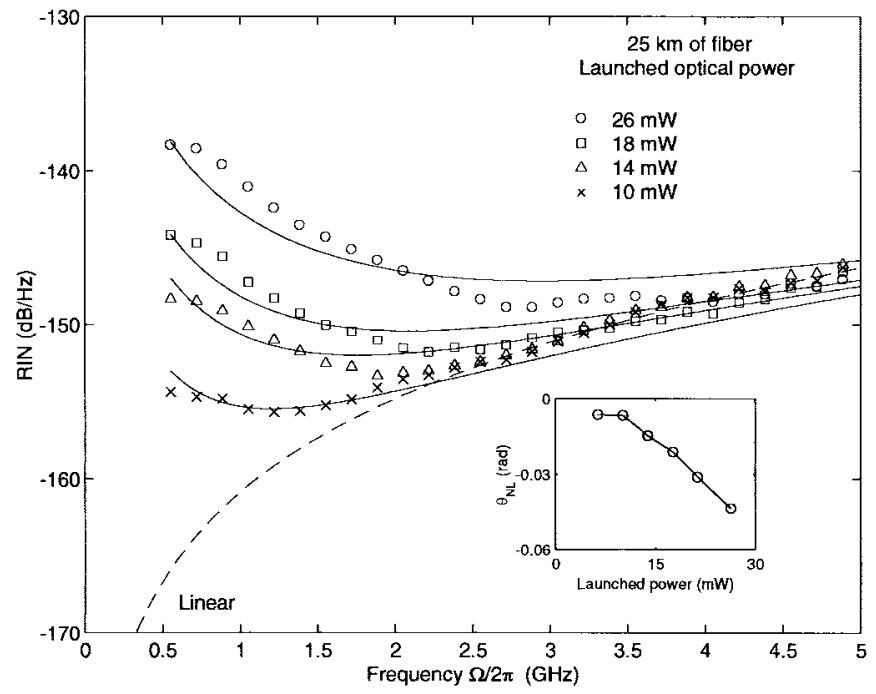

Fig. 5. RIN for $25 \mathrm{~km}$ of fiber and several launched powers into the fiber. Dashed line: RIN in the absence of nonlinear effects. Solid line is theory. The inset shows the nonlinear phase change as a function of launched power.

SBS backscattering of the incident field sidebands rather than beating of the small reflection of the incident field with itself.

The excess noise of the Stokes wave around $\Omega_{B}$ [Fig. 6(d)] can be well explained as originating from mixing of the Stokes wave with a small reflection of the modulated incident wave caused by Rayleigh scattering. Thus, the complex electric field amplitude of the backward propagating wave at the fiber input, $\tilde{E}_{B}$, can be expressed approximately as:

$$
\tilde{E}_{B}=P_{S}^{1 / 2}+\gamma_{R} \bar{P}_{0}^{1 / 2} \sum_{n} c_{n} e^{\mathrm{in}\left(\Omega t+\varphi_{\mathrm{FM}}\right)} e^{i \Omega_{B} t}
$$

where $P_{S}$ is the backreflected Stokes power at the fiber input, $\bar{P}_{0}$ is the integrated power of the forward propagating field along the fiber, and $\gamma_{R}$ is the field reflection coefficient due to Rayleigh scattering. The RIN power (RINP) at $\Omega_{B}+n \Omega$ can be determined experimentally by integrating the RIN over the spectral width of the spike (or by normalizing the RIN by the value at $0 \mathrm{~Hz}$ ). From (13), we find that the RINP of the backward propagating field, $\mathrm{RINP}_{B}$, is proportional to $\left|c_{n}\right|^{2}$, that is:

$$
\operatorname{RINP}_{B}\left(\Omega_{B}+n \Omega\right)=2 \frac{\bar{P}_{0}}{P_{S}}\left|\gamma_{R}\right|^{2}\left|c_{n}\right|^{2} .
$$

Fig. 7 shows the factor $\left|\gamma_{R}\right|^{2}\left|c_{n}\right|^{2}$ determined from the experimentally measured $\operatorname{RINP}_{B}$ and (14). The value of $\bar{P}_{0}$ was estimated by assuming an exponential decay of the forward propagating field. By comparing this with $\left|c_{n}\right|^{2}$ from (8) (dotted line in Fig. 7), we obtain $\left|\gamma_{R}\right|^{2}=-35.5 \mathrm{~dB}$, which agrees well with the value expected for Rayleigh scattering.

\section{Explanation of SBS-Induced Phase Change}

In this section, it will be theoretically demonstrated that due to waveguiding of the acoustic wave in the optical fiber, the Brillouin gain is complex. The imaginary part of this gain shifts the phase of the carrier relative to the modulation sidebands when these do not undergo SBS. 


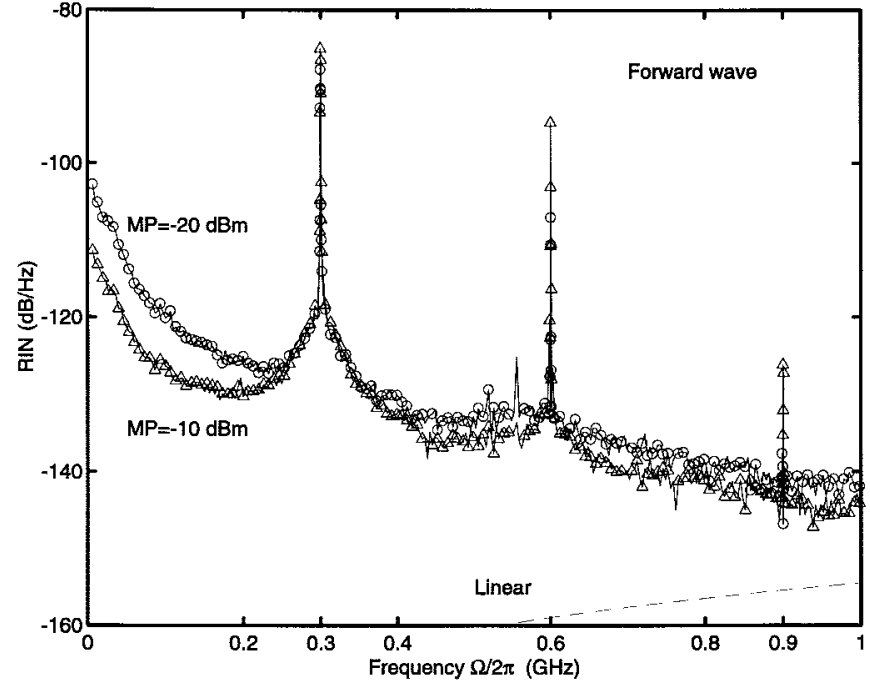

(a)

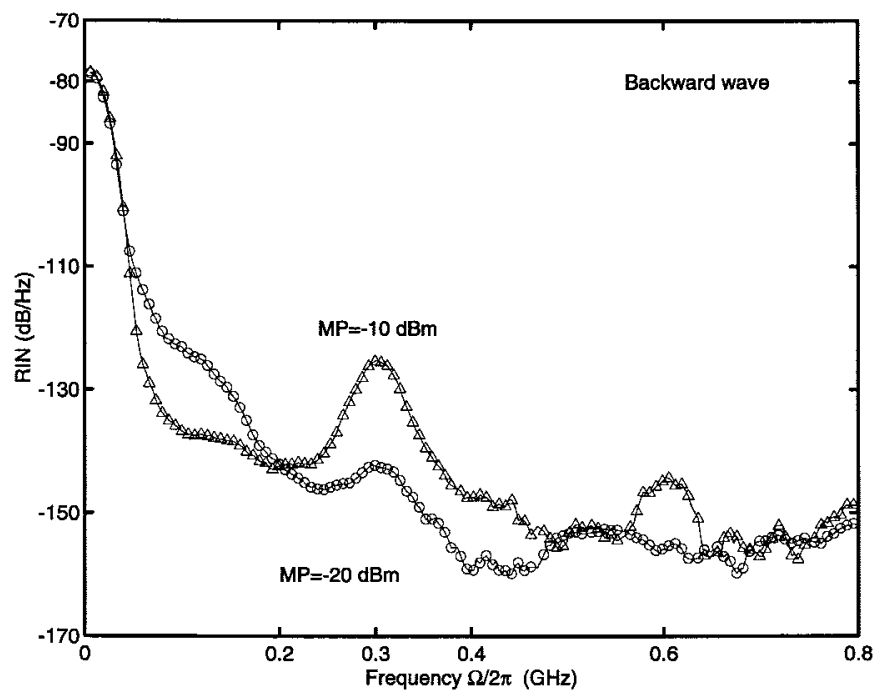

(c)

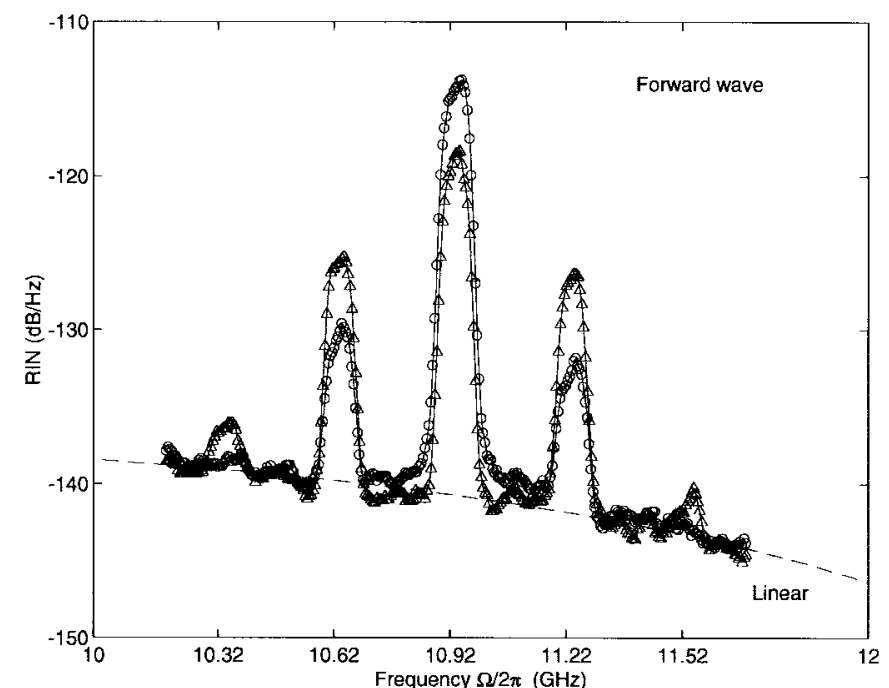

(b)

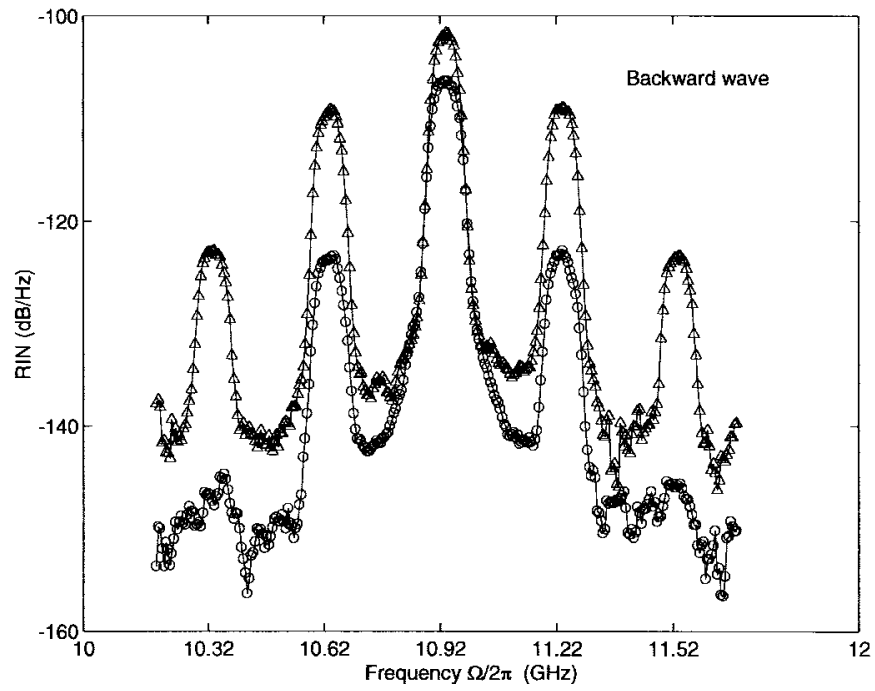

(d)

Fig. 6. RIN of (a), (b) forward and (c), (d), backward propagating waves after $50 \mathrm{~km}$ of fiber at (a), (c), low frequencies and (b), (d) around $\Omega_{B}$. The laser light was directly modulated at $300 \mathrm{MHz}$ with modulation powers (MP) $-20 \mathrm{dBm}$ (circles) and $-10 \mathrm{dBm}$ (triangles).

The theory of the acoustooptic interaction in an isotropic free cylinder is more fully developed in Appendix I. There we show that, in the case of backward Brillouin scattering, the acoustic wave can be well described by a material density fluctuation $\rho$ that satisfies the wave equation driven through electrostriction by the electric field $\mathbf{E}$, that is,

$$
\frac{\partial^{2} \rho}{\partial t^{2}}=v_{l}^{2}\left(1+\Gamma \frac{\partial}{\partial t}\right) \nabla^{2} \rho-\frac{\gamma}{2} \nabla^{2} E^{2}
$$

where $v_{l}$ is the longitudinal sound velocity, $\Gamma$ is the damping factor, and $\gamma$ quantifies the strength of the electrostrictive effect. This acoustic wave originates a nonlinear polarization $\mathbf{P}^{\mathrm{NL}}$ given by

$$
\mathbf{P}^{\mathrm{NL}}=\frac{\gamma}{\rho_{0}} \rho \mathbf{E}
$$

where $\rho_{0}$ is the material density.

In the absence of electric field, only a discrete set of acoustic modes $\rho_{n}(r, C)$ can propagate in the fiber. Neglecting the damping factor, these modes satisfy

$$
\left(\frac{i \Omega}{v_{l}}\right)^{2} \rho_{n}=\left[\nabla_{t}^{2}+k_{n}^{2}\right] \rho_{n} .
$$

Here the operator $\nabla_{t}^{2}$ is the Laplacian acting on the transverse coordinates, and $k_{n}$ are the eigenvalues or longitudinal propagation constants of the acoustic modes.

Only acoustic modes with no azimuthal variation will excite a polarization in a single-mode fiber. These are usually referred to as dilatational or longitudinal acoustic modes [19], [20] and are given by

$$
\rho_{n}(r)=\frac{1}{\left(\pi a_{c l}^{2}\right)^{1 / 2}} \frac{J_{0}\left(k_{l n} r\right)}{\left(J_{0}^{2}\left(k_{l n} a_{c l}\right)+J_{1}^{2}\left(k_{l n} a_{c l}\right)\right)^{1 / 2}}
$$

where $a_{c l}$ is the radius of the acoustic guide, which is usually the fiber cladding radius, and the eigenfunctions are normalized such that $\left\langle\rho_{n}, \rho_{n}\right\rangle=1$ (see Appendix II). The value of the transverse propagation constant of the longitudinal 


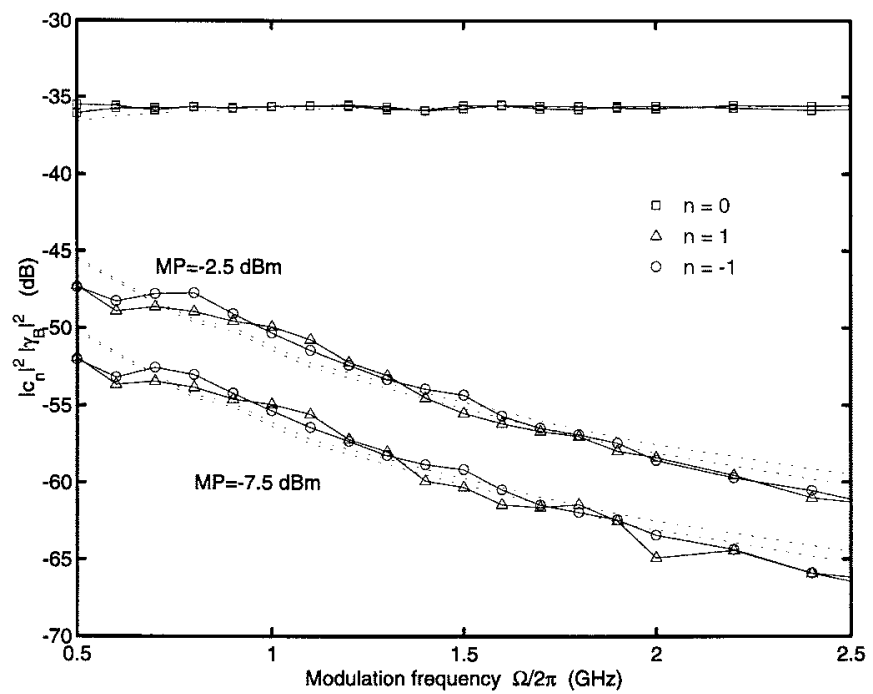

Fig. 7. Factor $\left|\gamma_{R}\right|^{2}\left|c_{n}\right|^{2}$ derived from the measured RIN power of the backward propagating field at the fiber input at $\Omega_{B}+n \Omega$ as a function of modulation frequency $\Omega / 2 \pi$ for two different MP's after $75 \mathrm{~km}$ of fiber. The dashed line is obtained with $\left|\gamma_{R}\right|^{2}=-35.5 \mathrm{~dB}$ and $\left|c_{n}\right|^{2}$ as determined from the laser chirp and modulation power.

acoustic modes $k_{l n}$ follows from application of boundary conditions. If we assume that the cylindrical surface is free of traction, i.e., $\boldsymbol{\tau} \cdot \mathbf{n}=0$, the Pochhammer frequency equation [20] is derived, from which $k_{n}$ and $k_{l n}=\sqrt{\left(\Omega / v_{l}\right)^{2}-k_{n}^{2}}$ can be calculated. When the particle vibrates in a longitudinal mode, radial and axial motions are coupled, and, contrary to what has been previously stated [7], [8], the eigenfunctions (18), even though they form a complete set, are not orthogonal.

Let us assume harmonically varying forward and backward propagating optical waves, that is,

$$
\begin{aligned}
\mathbf{E}(r, z, t)= & \frac{1}{\sqrt{2}}\left[a_{1}(z, t) e^{i\left(\omega_{1} t-\beta_{1} z\right)} \hat{\mathbf{e}}_{1}+a_{2}(z, t)\right. \\
& \left.\cdot e^{i\left(\omega_{2} t+\beta_{2} z\right)} \hat{\mathbf{e}}_{2}\right] E_{0}(r)+\text { c.c. }
\end{aligned}
$$

where $E_{0}(r)$ is the transverse distribution of the fundamental mode in a single-mode optical fiber and $\hat{\mathbf{e}}_{1}$ and $\hat{\mathbf{e}}_{2}$ are unit vectors in the directions of the forward and backward propagating fields, respectively. Here, we neglect fiber birefringence (see Appendix I for a discussion on birefringence induced by the acoustic wave) and assume linearly polarized electric fields, both along the same direction. The beating of these two fields gives rises to an acoustic wave at frequency $\Omega=\omega_{1}-\omega_{2}$ and with wavevector $k=\beta_{1}+\beta_{2} \approx 4 \pi n / \lambda_{0}$, where $\lambda_{0}$ is the optical wavelength and $n$ is the fiber modal index. Since the radius of the acoustic guide $a_{\mathrm{cl}}$ is much larger than the acoustic wavelength $\sim\left(\lambda_{0} / 2 n\right)$, a large number of acoustic modes are excited. The acoustic modes form a complete set and. therefore, the acoustic wave can be expressed as a linear combination of the $\rho_{n}(r, \varphi)$ such as

$$
\begin{aligned}
\rho(r, \varphi, z, t)= & a_{1}(z, t) a_{2}^{*}(z, t) \sum_{n} b_{n}(z, t) \rho_{n}(r, \varphi) \\
& \cdot e^{i(\Omega t-k z)}+\text { c.c. }
\end{aligned}
$$

Inserting the expansion for the acoustic wave (20) into the density wave (15) and using (17), we can obtain the coefficients $b_{n}(z, t)$ in a "least-squared" sense by solving the following system of linear differential equations:

$$
\begin{aligned}
\sum_{n} & {\left[\left\{v_{l}^{2}(1+i \Omega \Gamma)\left(k_{n}^{2}-k^{2}-2 i k \frac{\partial}{\partial z}\right)-i \Omega^{3} \Gamma\right\}\right.} \\
& \left.-2 i \Omega \frac{\partial}{\partial t}\right] b_{n}\left\langle\rho_{n}, \rho_{m}\right\rangle \\
= & -\frac{\gamma}{2} k^{2}\left\langle E_{0}^{2}, \rho_{m}\right\rangle .
\end{aligned}
$$

In writing (21), we have neglected derivatives of the electric field envelope amplitudes $a_{1}$ and $a_{2}$, second derivatives of $b_{n}$, and transverse derivatives of the electric field against longitudinal derivatives, and we have assumed that the modulation frequency of the acoustic wave envelope is much smaller than $\Omega$. Analytical expressions for the inner products in (21) have been found and are included in Appendix II (these integrals appeared in a similar context in [7] and [8] and were evaluated numerically). In practice, only modes with $k_{n} \approx k$ will contribute significantly to the mode expansion (20), and only a reduced number of coefficients $b_{n}$ is needed to perfectly describe the acoustic wave.

The evolution of the electric field envelope amplitudes can be found by substituting the electric field expansion (19) and the nonlinear polarization (16) into the optical wave equation. We obtain

$$
\begin{aligned}
\frac{\partial a_{1}}{\partial z}+ & v_{g} \frac{\partial a_{1}}{\partial t}+\frac{i}{2} \beta^{\prime \prime} \frac{\partial^{2} a_{1}}{\partial t^{2}} \\
= & \frac{\alpha}{2} a_{1}-\frac{g_{B}}{2}\left|a_{2}\right|^{2} a_{1} \\
& -\frac{\partial a_{2}}{\partial z}+v_{g} \frac{\partial a_{2}}{\partial t}+\frac{i}{2} \beta^{\prime \prime} \frac{\partial^{2} a_{2}}{\partial t^{2}} \\
= & \frac{\alpha}{2} a_{2}+\frac{g_{B}^{*}}{2}\left|a_{1}\right|^{2} a_{2} \\
g_{B}= & i \frac{\omega \gamma}{2 \rho_{0}} \sum_{n} b_{n}\left\langle\rho_{n}, E_{0}^{2}\right\rangle
\end{aligned}
$$

where $\omega \cong \omega_{1} \cong \omega_{2}, \beta \cong \beta_{1} \cong \beta_{2}, v_{g}$ is the fiber group velocity, $\beta^{\prime \prime}$ is the fiber dispersion parameter, and $\alpha$ is the fiber loss. The electric field is normalized to $\left\langle E_{0}, E_{0}\right\rangle=\omega \mu / \beta$ (see Appendix II), so that the power in each optical field is given by $\left|a_{i}\right|^{2}$.

From (22), we find that the real part of $g_{B}$ is the power Brillouin gain, whereas the imaginary part of $g_{B}$ is the origin of a phase modulation of the optical field. In steady state, the pump wave undergoes a phase change $\Delta \varphi_{1}$ given by

$$
\Delta \varphi_{1}(z)=-\frac{\operatorname{Im}\left\{g_{B}\right\}}{2} \int_{0}^{z}\left|a_{2}\right|^{2} d z .
$$

To a first approximation, we can neglect spatial derivatives of $b_{n}$ and can set $\left\langle\rho_{n}, \rho_{m}\right\rangle \approx 0$ for $n \neq m$. In the steady state, the coefficients $b_{n}$ are thus given by

$$
b_{n} \cong-\frac{\gamma}{2} \frac{k^{2}\left\langle E_{0}^{2}, \rho_{n}\right\rangle}{v_{l}^{2}(1+i \Omega \Gamma)\left(k_{n}^{2}-k^{2}\right)-i \Omega^{3} \Gamma} .
$$

Under this approximation, we find

$$
\begin{aligned}
g_{B}= & g_{B 0} \sum_{n} \frac{e^{-\left(k_{l n} w\right)^{2} / 2}}{\pi a_{c l}^{2}\left[J_{0}^{2}\left(k_{l n} a_{c l}\right)+J_{1}^{2}\left(k_{l n} a_{c l}\right)\right]} \\
& \cdot \frac{-i \Omega_{B}^{3} \Delta \Omega}{\left(k_{n}^{2} v_{l}^{2}-\Omega_{B}^{2}\right)\left(\Omega_{B}^{2}+i \Omega \Delta \Omega\right)-i \Omega^{3} \Delta \Omega}
\end{aligned}
$$




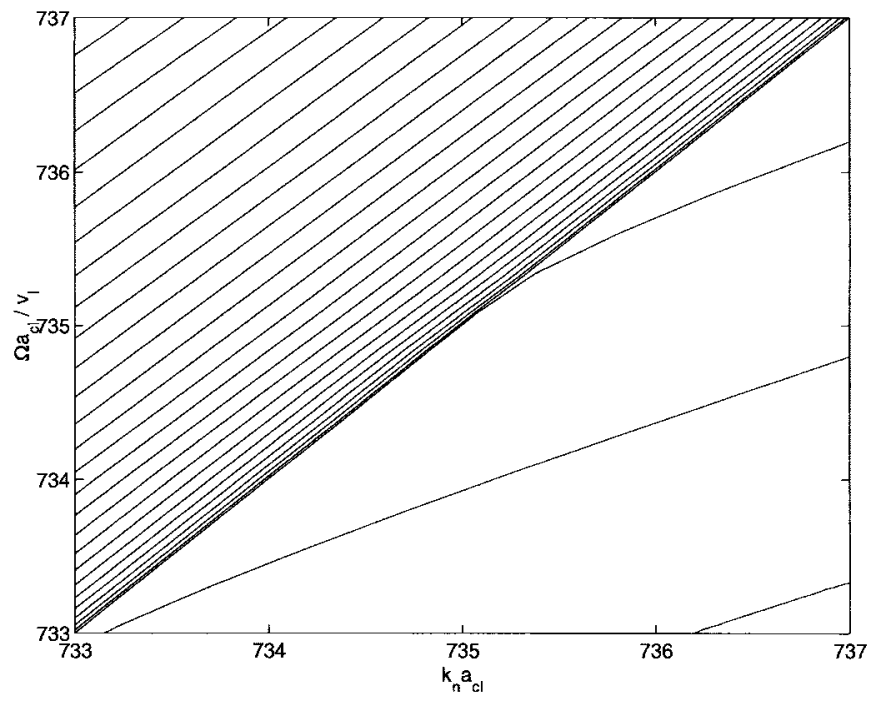

Fig. 8. Dispersion map of longitudinal acoustic modes for $v_{l} / v_{t}=1.6032$. For $\Omega_{l} / k_{n} \geq v_{l}$, the group velocity of the mode tends to the longitudinal sound velocity $v_{l}$ from above, whereas when $\Omega_{l} / k_{n}<v_{l}$ the mode becomes a surface wave and the group velocity approaches the transverse sound velocity $v_{t}$.

where $\Omega_{B}=k v_{l}, \Delta \Omega=\Gamma \Omega_{B}^{2}$, and $g_{B 0}=$ $\mu \omega^{2} k \gamma^{2} / 2 \beta \rho_{0} v_{l} \Delta \Omega=4 \pi^{2} n^{7} p_{12}^{2} / c \rho_{0} \lambda_{0}^{2} v_{l} \Delta \Omega$ are the approximate acoustic wave frequency, width, and maximum gain of the Brillouin spectrum in the absence of waveguiding effects, i.e., for $k v_{l} \approx \Omega \approx \Omega_{B}$.

As stated above, the dispersion map for the acoustic modes, i.e., the variation of the longitudinal propagation constant of the acoustic modes $k_{n}$ with the frequency $\Omega$, can be obtained by solving the Pochhammer frequency equation [20]. Here we only consider modes with real $k_{n}$, since modes with imaginary $k_{n}$ will attenuate very rapidly. On the contrary, the transverse propagation constants $k_{l n}$ can be real or purely imaginary, depending on whether the phase velocity $\Omega / k_{n}$ is greater or smaller than the longitudinal sound velocity $v_{l}$. The latter case corresponds to surface waves, which have maximum amplitudes near the surface of the cylinder. These surface waves will be hardly excited, since the optical mode is concentrated near the core region. Similarly, when the magnitude of $k_{l n}$ is large, the transverse profile of the acoustic mode becomes highly oscillatory and the overlap integral between the optical field and the acoustic mode is very small. Thus, only modes with $k_{n} \approx k$ and $\Omega / k_{n} \approx v_{l}$ will interact significantly with the optical field.

Numerical calculations have been performed to obtain the Brillouin gain for standard single-mode fiber with $a_{c l}=62.5$ $\mu \mathrm{m}$ and at optical wavelength $\lambda_{0}=1.55 \mu \mathrm{m}$. The dispersion map in the region $\Omega a_{c l} / v_{l} \approx k a_{c l} \approx 735$ is shown in Fig. 8 for $v_{l} / v_{t}=1.6032$. The normalized overlap integral between the electric field and the acoustic modes, $\beta /(\omega \mu) a_{c l}\left\langle E_{0}^{2}, \rho_{n}\right\rangle$, is plotted in Fig. 9 for several normalized frequencies. The asymmetry is due to the presence of surface waves.

If the effect of waveguiding of the acoustic wave is ignored, the imaginary part of the Brillouin gain at the frequency at which the real part reaches its maximum value is given by

$$
\operatorname{Im}\left\{g_{B}\right\}_{\max }=\operatorname{Re}\left\{g_{B}\right\}_{\max } \frac{\Delta \Omega}{4 \Omega_{B}} .
$$

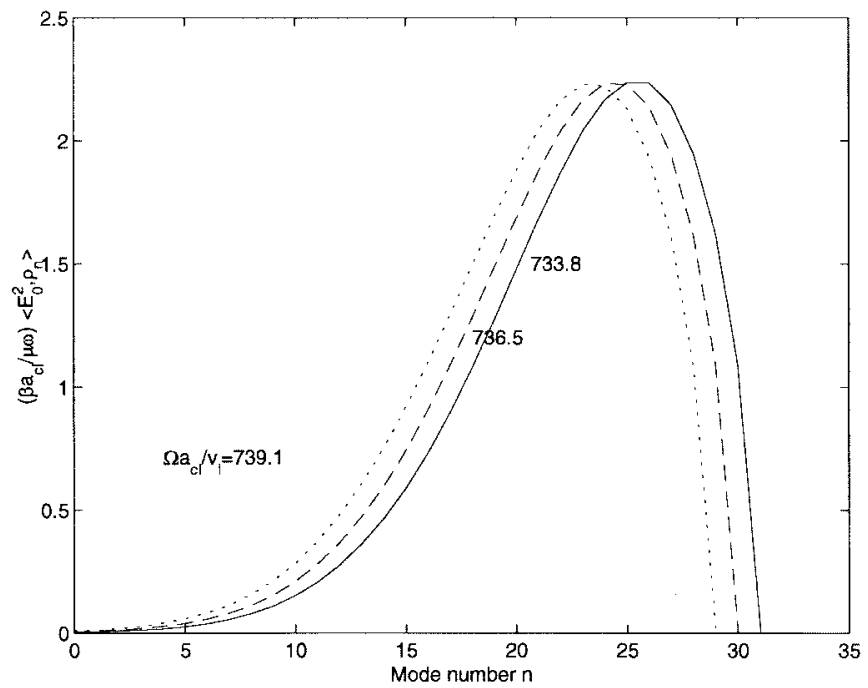

Fig. 9. Normalized overlap integral between the optical and acoustic modes $(\beta / \omega \mu) a_{c l}\left\langle E_{0}^{2}, \rho_{n}\right\rangle$ as a function of the (arbitrary) mode number $n$ for three different values of the normalized frequency $\omega_{l} a_{c l} / v_{l}$. The asymmetry is due to the low overlapping between the optical mode and the surface waves.

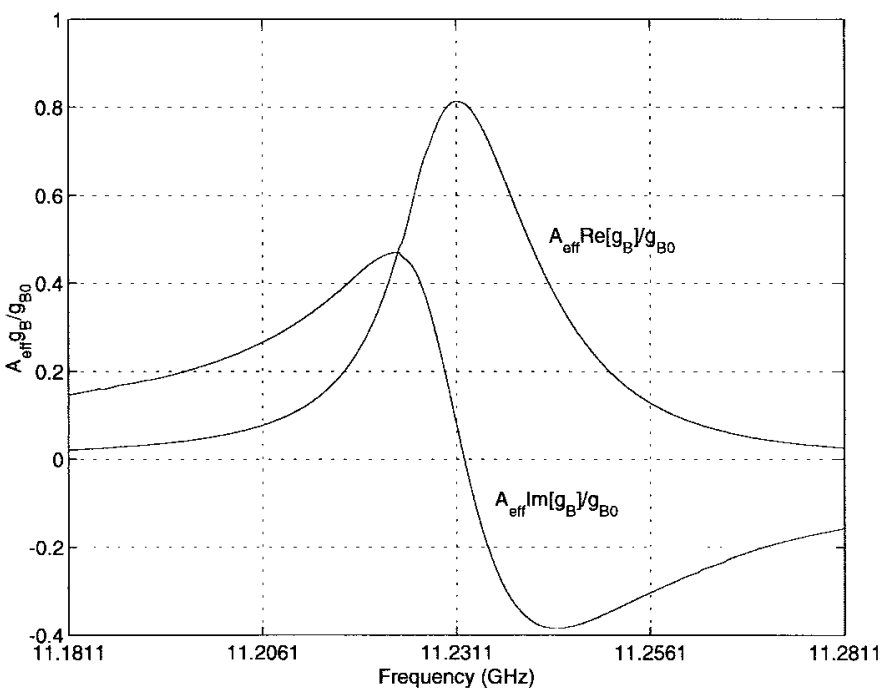

Fig. 10. Real and imaginary parts of the normalized gain $g_{B} A_{\text {eff }} / g_{B 0}$ as a function of frequency for $v_{l} / v_{t}=1.6032$.

Since $\Delta \Omega \ll \Omega_{B}$, this is very small. However, when (24) is used, due to the low coupling to the surface waves, $\operatorname{Re}\left\{g_{B}\right\}$ becomes slightly asymmetric, and $\operatorname{Im}\left\{g_{B}\right\}_{\max }$ is no longer negligible. Fig. 10 shows the real and imaginary parts of $g_{B} A_{\mathrm{eff}} / g_{B 0}$, where $A_{\mathrm{eff}}$ is the fiber core effective area, for $\Delta \Omega=15 \mathrm{MHz}$. If waveguiding effects are neglected, $\left.\left(\operatorname{Im}\left\{g_{B}\right\} / \operatorname{Re}\left\{g_{B}\right\}\right)\right|_{\max } \approx 3 \times 10^{-4}$, whereas here we obtain $\left.\left(\operatorname{Im}\left\{g_{B}\right\} / \operatorname{Re}\left\{g_{B}\right\}\right)\right|_{\max } \approx 0.1$. Using $g_{B 0} / A_{\text {eff }} \approx 0.06$ $\mathrm{mW}^{-1} \cdot \mathrm{km}^{-1}$ and $\int_{0}^{L}\left|a_{2}\right|^{2} d z \approx\left|a_{2}(0)\right|^{2} L_{\mathrm{eff}} / 2$, where $\left|a_{2}(0)\right|^{2}$ is the backscattered power and $L_{\text {eff }}$ is the fiber effective length, in (25), the dotted trace in Fig. 3 is obtained for the nonlinear phase shift. Thus, it can be concluded that this effect has the correct sign and magnitude to produce the nonlinear phase change experimentally measured. Moreover, the slight asymmetry in the $\operatorname{Re}\left\{g_{B}\right\}$ has been observed in our RIN measurements in Section IV, as well as in more precise 
measurements of the gain spectrum found in the literature (see, e.g., [9, Fig. 4(a)]).

The gain spectrum depends on fiber type [9], [14] and, from the above discussion, we expect that the induced phase shift will also exhibit a dependence. Experimental verification of the effect of the fiber type on induced phase shift will be the subject of future investigation.

\section{CONCLUSIONS}

The effect of SBS on MR and RIN was studied. It was shown experimentally that an SBS-induced phase change of the optical carrier can contribute to degradation of the performance of lightwave communication systems through an increase in the RIN and reduced linearity of the MR at low frequencies. This phase change was theoretically explained by including the waveguiding effect of the optical fiber on the acoustic wave. In addition, excess noise at harmonics of the acoustic frequency has been measured.

\section{APPENDIX I}

\section{THEORY OF THE ACOUSTOOPTIC INTERACTION}

A solid can become strained when placed in an electric field. In isotropic materials such as glass, the linear effect vanishes, and quadratic effects have to be considered. On the other hand, certain materials develop an electric moment under stress and, as a consequence, the dielectric constant is a function of the strain. In this case, the linear effect does not vanish and is the first-order effect. Physically, reversing the sign of the strain changes the state of the solid from tension to compression and so, in general, modifies the dielectric constant, whereas reversing the sign of the electric field does not change the physical situation, and the refractive index and strain should remain essentially unaltered.

The acoustic field variables that characterize particle motion and deformation in a vibrating material medium are the particle displacement vector $\mathbf{u}(\mathbf{r}, t)$, the strain tensor $\boldsymbol{\sigma}(\mathbf{r}, t)$, and the elastic restoring force or stress tensor $\tau(\mathrm{r}, t)$. These three field variables are related by the equation of motion [19], [20]

$$
\nabla \cdot \boldsymbol{\tau}=\rho_{0} \frac{\partial^{2} \mathbf{u}}{\partial t^{2}}
$$

as well as the strain-displacement relation

$$
\boldsymbol{\sigma}=\nabla_{s} \mathbf{u}
$$

and the elastic constitutive equation or Hooke's law, which, for a nonpiezoelectric material (such as an isotropic material), is given by

$$
\boldsymbol{\tau}=\mathbf{c}: \boldsymbol{\sigma}+\boldsymbol{\eta}: \frac{\partial \boldsymbol{\sigma}}{\partial t}+\frac{1}{2} \boldsymbol{\gamma}:\left(\mathbf{E} \cdot \mathbf{E}^{t}\right)
$$

Here, $\rho_{0}$ is the material density, $\mathbf{c}$ is the elastic stiffness tensor, $\eta$ is the viscosity tensor, which accounts for the existence of dissipative or frictional forces [21], $\gamma$ is the electrostriction tensor, which describes the stress resulting from the electric field $\mathbf{E}$, and the operator $\nabla_{s}$ takes the symmetric part of the displacement gradient, i.e.,

$$
\sigma_{i j}=\frac{1}{2}\left(\frac{\partial u_{i}}{\partial x_{j}}+\frac{\partial u_{j}}{\partial x_{i}}\right)=\sigma_{j i}
$$

The change in optical dielectric constant tensor $\varepsilon$ induced by the acoustic wave is given by

$$
\Delta\left(\frac{\varepsilon_{0}}{\varepsilon}\right)=\mathbf{p}: \sigma
$$

where $\mathbf{p}$ is the strainoptic tensor. It can be shown that the tensors $\boldsymbol{\gamma}$ and $\mathbf{p}$ are proportional [22].

The form of the tensors that describe the acoustooptic interaction can be derived from considerations of the symmetry of the medium. In an isotropic material such as glass, $\varepsilon$ is described by just one parameter, which, under suitable axes, is the value in the diagonal. On the other hand, the fourth rank tensors $\mathbf{p}, \boldsymbol{\eta}$, and $\boldsymbol{\gamma}$ have all the same form, and each of them can be reduced to just two constants. For the stiffness tensor, these are the Lamé's constants $\lambda$ and $\mu$. By substituting these tensors into (31) and (32), simplified expressions for the acoustic equation of motion (29) and the nonlinear polarization $\mathrm{P}^{\mathrm{NL}}$ are obtained as

$$
\begin{aligned}
\rho_{0} \frac{\partial^{2} \mathbf{u}}{\partial t^{2}}= & {\left[\rho_{0} v_{l}^{2}+\eta_{11} \frac{\partial}{\partial t}\right] \nabla(\nabla \cdot \mathbf{u}) } \\
& -\left[\rho_{0} v_{t}^{2}+\eta_{44} \frac{\partial}{\partial t}\right] \nabla \times \nabla \times \mathbf{u} \\
& +\frac{\gamma_{12}}{2} \nabla E^{2}+\gamma_{44} \nabla \cdot\left(\mathbf{E} \cdot \mathbf{E}^{T}\right) \\
\mathbf{P}^{\mathrm{NL}}= & -\gamma_{12}(\nabla \cdot \mathbf{u}) \mathbf{E}-2 \gamma_{44}\left(\nabla_{s} \mathbf{u}\right) \cdot \mathbf{E}
\end{aligned}
$$

where $v_{l}^{2}=(\lambda+2 \mu) / \rho_{0}$ and $v_{t}^{2}=\mu / \rho_{0}$ are the squared longitudinal and transverse sound velocities, respectively.

The electrostriction constants are given by [22]

$$
\begin{aligned}
\gamma_{12} & =n^{4} \varepsilon_{0} p_{12} \\
\gamma_{44} & =n^{4} \varepsilon_{0} p_{44}=n^{4} \varepsilon_{0} \frac{1}{2}\left(p_{11}-p_{12}\right)
\end{aligned}
$$

where $p_{11}=0.121$ and $p_{12}=0.270$ are the strain-optic coefficients for fused quartz, and $n$ is the refractive index.

The problem described by (33) and (34) is quite involved but can be greatly simplified by neglecting transversal versus longitudinal variations of the material displacement and the electric field, which is a valid approximation for the case of backward Brillouin scattering considered here in a single-mode optical fiber. As shown below, in this case, it is sufficient to consider the effect of $\gamma_{12}$, neglecting the contribution of $\gamma_{44}$, and we can express the equation of motion in terms of the material density variation $\rho$, which, in analogy with hydrodynamics, is defined as

$$
\rho=-\rho_{0} \nabla \cdot \mathbf{u}
$$

Substituting $\rho$ into (33) and (34), the acoustic wave equation for the material density fluctuation (15) is derived, where $\gamma=\gamma_{12}$ and $\gamma=\eta / \rho_{0}$

Now we outline the procedure in the general case, when the contribution of $\gamma_{44}$ is included. In order to determine which acoustic modes are excited by the electric field, we have to evaluate $\nabla E^{2}$ and $\nabla \cdot\left(\mathbf{E} \cdot \mathbf{E}^{T}\right)$. The terms oscillating at $\mu$ are given as shown in (37) and (38), at the bottom of the next page, where the three vector components are directed along $\hat{\mathbf{r}}, \hat{\varphi}$, and $\hat{\mathbf{z}}$. Thus, the electric field will excite acoustic 
modes with no azimuthal variation $\mathbf{u}_{0 n}$ and modes varying sinusoidally as $2 \varphi, \mathbf{u}_{2 n}$.

Next we analyze the nonlinear polarization (34) to determine which acoustic modes can excite the fundamental fiber mode. The necessary condition is that $\nabla \cdot \mathbf{u}$ and $\nabla_{s} \mathbf{u}$ have no azimuthal variation. For $\nabla \cdot \mathbf{u}$, this is only satisfied for modes $\mathbf{u}_{0 n}$. Let's examine now $\nabla_{s} \mathbf{u}$ as

$$
\begin{aligned}
\nabla_{s} \mathbf{u}= & \frac{1}{2}\left\{\left(\frac{\partial u_{r}}{\partial r}+\frac{u_{r}}{r}+\frac{1}{r} \frac{\partial u_{\varphi}}{\partial r}\right)(\hat{\mathbf{x}} \hat{\mathbf{x}}+\hat{\mathbf{y}} \hat{\mathbf{y}})+2 \frac{\partial u_{z}}{\partial r} \hat{\mathbf{z}} \hat{\mathbf{z}}\right. \\
& +\left(\frac{\partial u_{r}}{\partial r}-\frac{u_{r}}{r}-\frac{1}{r} \frac{\partial u_{\varphi}}{\partial r}\right)[\cos (2 \varphi)(\hat{\mathbf{x}} \hat{\mathbf{x}}-\hat{\mathbf{y}} \hat{\mathbf{y}}) \\
& +\sin (2 \varphi)(\hat{\mathbf{x}} \hat{\mathbf{y}}-\hat{\mathbf{y}} \hat{\mathbf{x}})] \\
& +\left(r \frac{\partial}{\partial r}\left(\frac{u_{\varphi}}{r}\right)+\frac{1}{r} \frac{\partial u_{r}}{\partial \varphi}\right)[\sin (2 \varphi)(-\hat{\mathbf{x}} \hat{\mathbf{x}} \\
& +\hat{\mathbf{y}} \hat{\mathbf{y}})+\cos (2 \varphi)(\hat{\mathbf{x}} \hat{\mathbf{y}}+\hat{\mathbf{y}} \hat{\mathbf{x}})] \\
& +\left(\frac{\partial u_{z}}{\partial r}+\frac{\partial u_{r}}{\partial z}\right)[\cos (\varphi) \hat{\mathbf{x}} \hat{\mathbf{z}}+\sin (\varphi) \hat{\mathbf{y}} \hat{\mathbf{z}}] \\
& \left.+\left(\frac{\partial u_{\varphi}}{\partial z}+\frac{\partial u_{z}}{\partial \varphi}\right)[-\sin (\varphi) \hat{\mathbf{x}} \hat{\mathbf{z}}+\cos (\varphi) \hat{\mathbf{y}} \hat{\mathbf{z}}]\right\} .
\end{aligned}
$$

From (39), it can be concluded that modes $\mathbf{u}_{0 n}$ in combination with factors in (39) with no azimuthal variation and modes $\mathbf{u}_{2 n}$ in combination with factors in (39) varying as $2 \varphi$ will excite the fundamental fiber mode.

The approximation in Section $\mathrm{V}$ is justified from (39) since the only terms from $\nabla_{s} \mathbf{u}$ that contribute to $\mathbf{P}_{\mathrm{NL}}$ are proportional to transverse variations of the material displacement. Similarly, only transverse derivatives of the electric field appear in the expression for $\nabla \cdot\left(\mathbf{E} \cdot \mathbf{E}^{T}\right)$ in (38).

The material displacement in the presence of excitation can be expanded in terms of the eigenmodes as we did in (20), and we can write

$$
\begin{aligned}
\mathbf{u}(r, \varphi, z, t)= & a_{1}(z, t) a_{2}^{*}(z, t)\left[\sum_{n} c_{n}(z, t) \mathbf{u}_{0 n}(r, \varphi)\right. \\
& \left.+d_{n}(z, t) \mathbf{u}_{2 n}(r, \varphi)\right] e^{i(\Omega t-k z)}+\text { c.c. }
\end{aligned}
$$

A similar approach to that in Section $\mathrm{V}$ can be followed to determine the coefficients of this expansion.

Equations (34) and (39) show that, even though the material is isotropic, due to the acoustooptic interaction it becomes anisotropic and birefringent.

\section{APPENDIX II}

\section{SOME USEFUL INTEGRALS}

The inner product used in this paper is defined as

$$
\left\langle\rho_{n}, \rho_{m}\right\rangle=\int_{0}^{2 \pi} \int_{0}^{a} r \rho_{n}(r, \varphi) \rho_{m}^{*}(r, \varphi) d r d \varphi
$$

Using

$$
\begin{aligned}
\int_{0}^{1} r J_{v}(p r) J_{v}(q r) d r \\
= \begin{cases}\frac{1}{2}\left(J_{v}^{2}(p r)-J_{v+1}(p r) J_{v-1}(p r)\right), & \text { if } p=q \\
\frac{p J_{v+1}(p r) J_{v}(q r)-q J_{v}(p r) J_{v+1}(q r)}{p^{2}-q^{2}}, & \text { if } p \neq q\end{cases}
\end{aligned}
$$

we find (43), shown at the bottom of the page. If we use a Gaussian approximation for the fundamental fiber mode with mode beam waist $w, E_{0}(r)=\left(\omega \mu / \pi w^{2} \beta\right)^{1 / 2} \exp \left(-r^{2} / 2 w^{2}\right)$, where $E_{0}(r)$ is normalized so that $\left|a_{i}\right|^{2}$ equals the power carried by mode $i$, i.e., $\left\langle E_{0}, E_{0}\right\rangle=\omega \mu / \beta$, we find

$$
\begin{aligned}
\left\langle E_{0}^{2}, \rho_{m}\right\rangle & \cong 2 \pi \int_{0}^{\infty} r E_{0}^{2}(r) \rho_{m}(r) d r \\
& =\frac{\omega \mu}{\beta} \frac{e^{-\left(\left[k_{l m} w\right] / 2\right)^{2}}}{\left(\pi a_{c l}^{2}\right)^{1 / 2}\left[J_{0}^{2}\left(k_{l m} a_{c l}\right)+J_{1}^{2}\left(k_{l m} a_{c l}\right)\right]^{1 / 2}}
\end{aligned}
$$

\section{ACKNOWLEDGMENT}

The authors are grateful to Ortel Corporation, in particular to Dr. J. Ianelli and Dr. T. R. Chen, for providing the lasers. They also want to thank Dr. W. Marshall and D. Provenzano for useful discussions.

$$
\begin{aligned}
\nabla E^{2}(r, z, t)= & a_{1}(z, t) a_{2}^{*}(z, t) \hat{\mathbf{e}}_{1} \cdot \hat{\mathbf{e}}_{2}^{*}\left[\begin{array}{c}
\frac{\partial E_{0}^{2}(r)}{\partial r} \\
0 \\
-i k E_{0}^{2}(r)
\end{array}\right] e^{i(\Omega t-k z)} \\
\nabla \cdot\left(\mathbf{E} \cdot \mathbf{E}^{T}\right)(r, \varphi, z, \Omega)= & \frac{1}{2} a_{1}(z, \Omega) a_{2}^{*}(z, \Omega) \frac{\partial E_{0}^{2}(r)}{\partial r} \\
& \cdot \exp (-i k z)\left\{\left[\begin{array}{c}
e_{1 x} e_{2 x}^{*}+e_{1 y} e_{2 y}^{*} \\
0 \\
0
\end{array}\right]+\left[\begin{array}{c}
\cos (2 \varphi)\left(e_{1 x} e_{2 x}^{*}-e_{1 y} e_{2 y}^{*}\right)+\sin (2 \varphi)\left(e_{1 x} e_{2 y}^{*}+e_{1 y} e_{2 x}^{*}\right) \\
\cos (2 \varphi)\left(e_{1 x} e_{2 x}^{*}+e_{1 y} e_{2 y}^{*}\right)+\sin (2 \varphi)\left(e_{1 x} e_{2 y}^{*}-e_{1 y} e_{2 x}^{*}\right) \\
0
\end{array}\right]\right\}
\end{aligned}
$$

$$
\left\langle\rho_{n}, \rho_{m}\right\rangle=2 \frac{k_{l n} J_{1}\left(k_{l n} a_{c l}\right) J_{0}\left(k_{l m} a_{c l}\right)-k_{l m} J_{0}\left(k_{l n} a_{c l}\right) J_{1}\left(k_{l m} a_{c l}\right)}{\left[k_{l n}^{2}-k_{l m}^{2}\right]\left[J_{0}^{2}\left(k_{l n} a_{c l}\right)+J_{1}^{2}\left(k_{l n} a_{c l}\right)\right]^{1 / 2}\left[J_{0}^{2}\left(k_{l m} a_{c l}\right)+J_{1}^{2}\left(k_{l m} a_{c l}\right)\right]^{1 / 2}}, \quad n \neq m
$$




\section{REFERENCES}

[1] A. R. Chraplyvy, "Limitation on lightwave communications imposed by optical fiber nonlinearities," J. Lightwave Technol., vol. 8, pp. 1548-1557, Oct. 1990.

[2] D. A. Fishman and J. A. Nagel, "Degradations due to stimulated Brillouin scattering in multigigabit intensity-modulated fiber-optic systems," J. Lightwave Technol., vol. 11, pp. 1721-1728, Nov. 1993.

[3] H. Yoshinaga, M. Kagami, K. Kikushima, and K. Suto, "Nonlinear distorsion due to stimulated Brillouin scattering and its suppression in SCM video-transmission systems," in Proc. OFC/IOOC, 1993, pp. 257-258.

[4] R. G. Smith, "Optical power handling capacity of low loss optical fibers as determined by stimulated Brillouin and Raman scattering," Appl. Opt., vol. 11, no. 11, pp. 2489-2492, 1972.

[5] J. Stone and A. R. Chraplyvy, "Spontaneous Brillouin noise in longdistance high-bandwidth optical-fiber transmission," Electron. Lett., vol. 19, no. 8, pp. 275-277, 1983.

[6] R. M. Shelby, M. D. Levenson, and P. W. Bayer, "Guided acousticwave Brillouin scattering," Phys. Rev. B, vol. 31, no. 8, pp. 5244-5252, Apr. 1985.

[7] E. M. Dianov, A. V. Luchnikov, A. N. Pilipetskii, and A. N. Starodumov, "Long-range interaction of soliton pulse trains in a single-mode fiber," Sov. Lightwave Commun., vol. 1, pp. 37-43, 1991.

[8] A. Fellegara and S. Wabnitz, "Electrostrictive cross-phase modulation of periodic pulse trains in optical fibers," Opt. Lett., vol. 23, no. 17, pp. 1357-1359, Sept. 1998.

[9] N. Shibata, R. G. Waarts, and R. P. Braun, "Brillouin gain spectra for single mode fibers having pure silica, $\mathrm{GeO}_{2}$ doped, and $\mathrm{P}_{2} \mathrm{O}_{5}$ doped cores," Opt. Lett., vol. 12, no. 4, pp. 269-271, Apr. 1987.

[10] E. Peral, W. K. Marshall, and A. Yariv, "Precise measurement of semiconductor laser chirp using effect of propagation in dispersive fiber and application to simulation of transmission through fiber gratings," $J$. Lightwave Technol., vol. 16, pp. 1874-1880, Oct. 1998.

[11] A. Royset, L. Bjerkan, D. Myhre, and L. Hafskjaer, "Use of dispersive optical fiber for characterization of chirp in semiconductor lasers," Electron. Lett., vol. 30, no. 9, pp. 710-712, Apr. 1994.

[12] B. Wedding, "Analysis of fiber transfer funcion and determination of receiver frequency response for dispersion supported transmission," Electron. Lett., vol. 30, no. 1, pp. 58-59, Jan. 1994.

[13] A. Cartaxo, B. Wedding, and W. Idler, "Influence of fiber nonlinearity on the phase noise to intensity noise conversion in fiber transmission: Theoretical and experimental analysis," J. Lightwave Technol., vol. 16, pp. 1187-1193, July 1998.

[14] M. Horowitz, A. R. Chraplyvy, R. W. Tkach, and J. L. Zyskind, "Broadband transmitted intensity noise indeuced by Stokes and anti-Stokes Brillouin scattering in single-mode fibers," IEEE Photon. Technol. Lett., vol. 9, pp. 124-126, Jan. 1997.

[15] E. Peral, W. K. Marshall, D. Provenzano, and A. Yariv, "Effect of many weak side modes on relative intensity noise of distributed feedback semiconductor lasers," Appl. Phys. Lett., vol. 72, no. 8, pp. 888-890, Feb. 1998.
[16] S. Wu and A. Yariv, "Theoretical and experimental investigation of conversion of phase noise to intensity noise by Rayleigh scattering in optical fibers," Appl. Phys. Lett., vol. 59, no. 10, pp. 1156-1158, Sept. 1991.

[17] A. D. Kersey and M. A. Davis, "Brillouin bandwidth determination from excess-noise characteristics of SBS signals in single-mode fibers," in Proc. OFC/IOOC, 1993, pp. 206-207.

[18] R. W. Boyd and K. Rzazewski, "Noise initiation of stimulated Brillouin scattering," Phys. Rev. A, vol. 42, no. 9, pp. 5514-5521, Nov. 1990.

[19] B. A. Auld, Acoustic Fields and Waves in Solids. Malabar, India: Krieger, 1990.

[20] J. D. Achenbach, Wave Propagation in Elastic Solids. Amsterdam, The Netherlands: North Holland, 1973.

[21] Landau and Lifshitz, Theory of Elasticity. Oxford, U.K.: Pergamon Press, 1986.

[22] J. F. Nye, Physical Properties of Crystals. Oxford, U.K.: Clarendon Press, 1957.

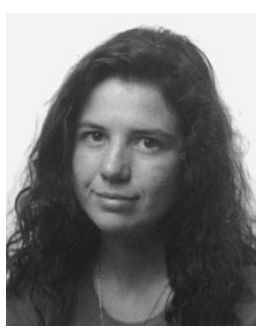

Eva Peral was born on November 23, 1972, in Granada, Spain. She received the degree of Ingeniero Superior de Telecomunicaciones with Honors from the Universidad Politécnica de Valencia (UPV), Spain, in 1995, the M.S. degree in electrical engineering from the California Institute of Technology (Caltech), Pasadena, in 1998, and the Ph.D degree in Ingeniería de Telecomunicaciones from UPV in 1998. She is currently working toward the Ph.D. degree in electrical engineering at Caltech.

During 1995, she was a visiting student at the University of Strathclyde,Glasgow, Scotland, and collaborated with the Optoelectronics Group where she was engaged in integrated optics. She has worked for Philips, Valencia, Spain, and Mercedes-Benz, Sindelfingen, Germany. She started her doctoral studies with the Optical Communications and Photonic Engineering Group at UPV. Since 1996, she has been at Caltech. Her research interests include semiconductor lasers, fiber gratings, linear and nonlinear propagation in optical fiber, and integrated optics.

Ms. Peral received the First National Award for Academic Excellency from the Spanish Ministry of Culture and Education in 1996.

Amnon Yariv (S'56-M'59-F'70-LF'95), for photograph and biography, see this issue, p. 1175. 